\title{
FREQUÊNCIA DE SÍFILIS EM GESTANTES
}

\section{FREQUENCY OF SYPHILIS IN PREGNANT WOMEN}

\author{
Karlene Kristina dos Santos ${ }^{1}$, Lara Ciríaco Alves ${ }^{1}$, Luiza Sousa Vilano1, \\ Nayani Abrantes Borges ${ }^{1}$, Juliana Pereira Soares ${ }^{2}$, Leandro Henrique Ávila Silveira², \\ Raphaela Pollyana Moura Nascimento ${ }^{3}$, Maria Clara Marangoni², \\ Juliana Barroso Zimmermmann ${ }^{1,2}$
}

\begin{abstract}
RESUMO
O cuidado primário tem um papel importante na prevenção e no tratamento de doenças infecciosas, pois no contexto gestacional algumas dessas doenças podem causar complicações graves ao recém-nascido, levando inclusive à morte fetal. Os autores apresentam um estudo transversal em que participaram 299 gestantes no Serviço Pré-natal em Juiz de Fora e 66 gestantes oriundas do serviço de obstetrícia da Faculdade de Medicina de Barbacena. A idade média foi de 24,60 anos. Houve sífilis em ambos os grupos, mas não houve casos de sífilis congênita. Os autores concluíram que a frequência de sífilis apresentou distribuição uniforme em ambos os serviços e sugerem medidas adequadas de controle e tratamento de gestantes.
\end{abstract}

Palavras-chave: Sífilis congênita; vaginose bacteriana; candidíase; infecções por tricomonas; mulheres grávidas

\begin{abstract}
Primary care has an important role in the prevention and treatment of infectious diseases since in the gestational context some of these diseases can cause severe complications to the newborn, including fetal death. This is a cross-sectional study assessing 299 pregnant women who attended the Pre-Natal Service in Juiz de Fora and 66 pregnant women who attended the Obstetrics Service of Barbacena School of Medicine. The average age was 24.60 years old. There was syphilis in both groups, but there were no cases of congenital syphilis. The authors concluded that the frequency of syphilis had a uniform distribution among both services and suggest adequate measures of control and treatment of pregnant women.
\end{abstract}

Keywords: Syphilis congenital; vaginosis bacterial; candidiasis; trichomonas infections; pregnant women

A atenção primária possui importante papel na prevenção e no tratamento de doenças infecciosas no contexto gestacional, já que algumas dessas doenças podem determinar não apenas desconforto materno mas também graves complicações ao recém-nascido, culminando inclusive com a morte fetal ${ }^{1}$.

A sífilis é uma doença infectocontagiosa causada pelo Treponema pallidum. A sua transmissão se dá por via sexual ou vertical. A transmissão vertical da sífilis - sífilis congênita (SC) - pode ocorrer em qualquer fase da gestação e em qualquer estágio da doença. Quanto mais recente for a infecção, maior será o risco de contaminação fetal grave ${ }^{2}$.

Como diretrizes definidas pelo Ministério da Saúde para controle da SC estão o controle da transmissão vertical da doença e o acompanhamento do comportamento da infecção nas gestantes, bem como avaliação e planejamento do tratamento, prevenção e controle. Sendo assim, a transmissão vertical da sífilis durante a gestação pode refletir falhas que variam desde a adesão da gestante ao pré-natal, não identificação da gestante de risco para a
Clin Biomed Res. 2018;38(1):81-86

1 Serviço de Obstetrícia, Faculdade de Medicina de Barbacena (FAME). Barbacena, MG, Brasil.

2 Serviço de Obstetrícia do HU/CAS, Universidade Federal de Juiz de Fora (UFJF). Juiz de Fora, MG, Brasil.

3 Prefeitura Municipal de Barbacena, Serviço de Saúde da Família. Barbacena, MG, Brasil.

Autor correspondente: Juliana Barroso Zimmermmann julianabz@uol.com.br Departamento Materno Infantil, Faculdade de Medicina, Universidade Federal de Juiz de Fora (UFJF) Av. Eugênio do Nascimento, s/n. 36038-330, Juiz de Fora, MG, Brasil. 
doença, não aderência do casal ao tratamento ou sua realização de forma inadequada e incompleta. Portanto, a SC é uma doença evitável, e mesmo um único caso representa uma falha do sistema público de saúde ${ }^{2,3}$.

No Brasil, a incidência de SC cresceu nos últimos anos. Segundo o Departamento de Informática do Sistema Único de Saúde (DATASUS), no ano de 2010 foram confirmados 6.917 casos, 1.577 casos a mais do que no ano de 2009. Sabe-se que o aumento do número de notificações pode representar não só o real aumento da incidência como também falhas nos pré-natais, tanto relacionadas à triagem da sífilis quanto a uma dificuldade em notificar a doença $a^{4,5}$. Apesar de os protocolos determinarem a triagem de sífilis na gestação e, sendo os testes positivos, o subsequente tratamento das gestantes e de seus parceiros, a incidência de sífilis tem crescido no Brasil, especialmente nos últimos anos, o que evidencia alguma falha na abordagem dessas mulheres. Além disso, recentemente verificou-se que a penicilina benzatina, medicação usada para o tratamento da mãe e do bebê, não estava sendo disponibilizada no mercado, em decorrência da dificuldade de produção do princípio ativo e de problemas na manufatura do medicamento, o que atrapalha o tratamento da gestante e eleva a frequência de $\mathrm{SC}^{6}$.

Baseados no exposto, nos propomos a avaliar a frequência de sífilis em gestantes atendidas pelos serviços de obstetrícia da Faculdade de Medicina de Barbacena e da Universidade Federal de Juiz de Fora.

\section{MÉTODOS}

\section{Pacientes}

Trata-se de um estudo de corte transversal no qual foram incluídas 365 gestantes, sendo 299 atendidas no Serviço de Pré-Natal da Universidade Federal de Juiz de Fora e 66 no Serviço de Obstetrícia da Faculdade de Medicina de Barbacena, Unidade Básica de Saúde Santa Cecília, totalizando 365 gestantes de baixo risco obstétrico. O cálculo amostral foi realizado considerando como população alvo os casos de sífilis em gestantes no Brasil no último ano, totalizando 15.247 gestantes, com nível de confiança de $90 \%$, erro amostral de $5 \%$, o que totalizou amostra de 266 gestantes. A escolha dos locais de atendimento foi baseada na possibilidade de uso da mesma ficha cadastral para atendimento da gestante ${ }^{7}$, o que permitiu a uniformização dos dados a serem pesquisados, bem como na boa avaliação de ambos os serviços de pré-natal, de acordo com o Índice de Acesso e Assistência ao Pré-natal (IAAPN), desenvolvido em recente estudo na Faculdade de Medicina de Barbacena. $\mathrm{Na}$ oportunidade, o serviço foi considerado como pré-natal adequado ${ }^{8}$. Foram excluídas pacientes que não quiseram participar do estudo e que não tinham ao menos um exame de venereal disease research (VDRL), independentemente do número de consultas realizadas. Incluíram-se gestantes que permitiram que seus dados fossem avaliados, gestantes matriculadas em regime de pré-natal em qualquer um dos serviços e que realizaram ao menos um exame de VDRL durante o pré-natal.

\section{Método Clínico}

Foram avaliados dados da anamnese (número de gestações, partos, abortos e consultas), bem como dados do exame físico (pressão arterial, peso, altura uterina, batimentos cardíacos fetais, toque obstétrico e avaliação de edemas). Em relação aos exames complementares, utilizou-se como propedêutica básica de pré-natal as orientações do Manual de Assistência Pré-Natal, que são: hemograma, glicemia de jejum, grupo sanguíneo $A B O$ e Rh, sorologia para toxoplasmose, rubéola, hepatite B (HBsAg), HIV (anti - HIV 1 e 2), VDRL, exame de urina (elementos anormais do sedimento [EAS] e urocultura $)^{8,9}$. Os exames foram realizados trimestralmente, de acordo com a necessidade, e seus resultados sorológicos foram anotados em ficha de pré-natal e cartão da gestante. Entretanto, para este estudo selecionaram-se os dados referentes a VDRL e fluorescent treponema antigen absorbent (FTA-ABS), quando necessário ${ }^{9,10}$.

\section{Laboratório}

Para o diagnóstico laboratorial de sífilis, utilizou-se - Manual Técnico de Pré-Natal e Puerpério do Ministério da Saúde ${ }^{10}$, que orienta a solicitação de um teste de VDRL na primeira consulta de pré-natal. Quando negativo, o exame foi repetido no segundo e terceiro trimestres de gravidez. Por outro lado, quando positivo fez-se o teste confirmatório FTA-ABS ${ }^{9,10}$.

\section{Método Estatístico}

Todos esses dados foram transmitidos para planilha eletrônica e processados em software STATA, versão 9.2. A partir das variáveis estudadas, foram produzidas tabelas compostas com frequência absoluta e relativa, onde foram calculadas medidas de posição, tendência central e dispersão. A existência de relação entre as variáveis foi definida por teste de qui-quadrado, exato Fischer, teste t ou Mann Whitney, conforme a variável estudada, para as devidas comparações entre os grupos. Considerou-se $p<0,05$. 


\section{RESULTADOS}

\section{Aspectos Clínicos e Epidemiológicos das Gestantes de Acordo com a Origem das Gestantes}

Os aspectos clínicos das pacientes são apresentados na Tabela 1. Não houve diferença na idade entre as pacientes atendidas em Juiz de Fora e em Barbacena $(p>0,05)$ mas houve diferença em relação às seguintes variáveis: número de consultas $(p=0,00)$, idade gestacional no início do pré-natal $(p=0,00)$, ganho de peso $(p<0,05)$, escolaridade $(p<0,05)$, idade gestacional no início do pré-natal $(p=0,0007)$ e cor da pele $(p=0,00)$, de forma que as pacientes de Juiz de Fora foram aquelas que mais frequentemente se declararam brancas, tinham mais frequentemente curso superior, ganharam mais peso no pré-natal e tiveram maior número de consultas.

Em relação ao exame ginecológico, que consistiu na avaliação da vulva, vagina e colo uterino, verificou-se que 175 pacientes $(48,2 \%)$ tinham exame normal, $66(18,2 \%)$ tinham exame alterado, e em 124 pacientes $(33,6 \%)$ o exame não foi realizado. A comparação entre os grupos permitiu identificar que Barbacena foi a cidade com maior número de exames alterados $(p=0,00001$; qui-quadrado $=30,47)$. A citologia foi colhida no pré-natal em 249 gestantes, sendo considerada normal em 140 pacientes $(38,4 \%)$ e alterada em $109(29,9 \%)$. Quando se comparou em relação às cidades, na cidade de Barbacena foi identificada a maior ausência de exames colpocitológico no pré-natal ( $p=0,000$; qui-quadrado $=113,61)$.

\section{A Frequência de Sífilis}

Não se identificou nenhum caso de sífilis primária ao exame físico ginecológico (identificação do cancro). Quando se avaliou a presença de sífilis através da pesquisa do VDRL e confirmação com FTA-ABS, identificaram-se cinco casos de sífilis, que representaram $1,4 \%$ de frequência de sífilis em gestantes, conforme Tabela 2.

Quando se associou dados clínicos e epidemiológicos de gestantes com e sem sífilis, não houve diferença em relação a idade, número de gestações, partos, abortos e consultas, idade gestacional na primeira consulta, mas houve associação entre sífilis e baixa escolaridade $(p<0,05)$. Não houve associação entre sífilis e alterações citológicas, hepatite $B$ ou hepatite C. Não houve caso de sífilis congênita. Todas as pacientes foram tratadas com penicilina benzatina e controladas pelo VDRL, conforme preconiza o Ministério da Saúde.

Tabela 1: Dados epidemiológicos, ginecológicos e obstétricos das pacientes estudadas, de acordo com a origem das pacientes (Barbacena ou Juiz de Fora).

\begin{tabular}{|c|c|c|c|c|}
\hline \multirow{2}{*}{ Dados avaliados } & \multicolumn{2}{|c|}{ Origem } & \multirow{2}{*}{ Valor de $p$} & \multirow{2}{*}{$X^{2} / T$} \\
\hline & Barbacena & Juiz de Fora & & \\
\hline \multicolumn{5}{|l|}{ Aspectos epidemiológicos } \\
\hline Idade, anos (média $\pm \mathrm{DP})$ & $24,45 \pm 7,40$ & $24,64 \pm 6,06$ & 0,82 & $\mathrm{~T}=0,22$ \\
\hline \multicolumn{5}{|l|}{ Escolaridade, $\mathrm{n}$} \\
\hline Analfabeto & 0 & 2 & \multirow{5}{*}{0,00} & \multirow{5}{*}{$X^{2}=123,29$} \\
\hline Ensino Fundamental & 10 & 104 & & \\
\hline Ensino Médio & 26 & 161 & & \\
\hline Ensino Superior & 3 & 31 & & \\
\hline Não informado & 24 & 0 & & \\
\hline \multicolumn{5}{|l|}{ Cor, $\mathrm{n}$} \\
\hline Branca & 1 & 31 & & \\
\hline Parda & 22 & 117 & 0,00 & $X^{2}=107,28$ \\
\hline Negra & 20 & 111 & & \\
\hline Não informaram & 27 & 36 & & \\
\hline \multicolumn{5}{|l|}{ Aspectos obstétricos (média $\pm D P$ ) } \\
\hline Idade gestacional na primeira consulta, meses & $11,7 \pm 6,0$ & $15,7 \pm 5,6$ & 0,00 & $X^{2}=0,97$ \\
\hline Peso inicial, $\mathrm{kg}$ & $66,80 \pm 15,01$ & $67,18 \pm 14,92$ & 0,95 & $X^{2}=0,95$ \\
\hline Peso final, $\mathrm{kg}$ & $75,09 \pm 14,86$ & $77,20 \pm 13,90$ & 0,33 & $\mathrm{~T}=0,97$ \\
\hline Ganho de peso, kg & $7,7 \pm 3,8$ & $10,2 \pm 4,7$ & 0,0007 & $\mathrm{~T}=0,97$ \\
\hline Número de gestações & $1,8 \pm 1,4$ & $1,8 \pm 1,19$ & 0,09 & $\mathrm{~T}=0,00$ \\
\hline Número de partos & $0,63 \pm 0,9$ & $0,69 \pm 1,0$ & 0,65 & $\mathrm{~T}=0,45$ \\
\hline Número de abortos & $0,20 \pm 0,5$ & $0,17 \pm 0,46$ & 0,67 & $\mathrm{~T}=0,67$ \\
\hline Partos vaginais prévios & $0,48 \pm 1,03$ & $0,47 \pm 0,86$ & 0,91 & $\mathrm{~T}=0,10$ \\
\hline Partos cesáreos prévios & $0,15 \pm 0,40$ & $0,22 \pm 0,54$ & 0,35 & $\mathrm{~T}=0,92$ \\
\hline
\end{tabular}

$\mathrm{DP}=$ desvio padrão; $\mathrm{X}^{2}$ = qui-quadrado. 
Tabela 2: Identificação de sífilis nas pacientes estudadas, de acordo com a origem das pacientes.

\begin{tabular}{cccc}
\hline & Barbacena & $\begin{array}{c}\text { Juiz de } \\
\text { Fora }\end{array}$ & Total \\
\hline Sífilis (+), $\mathrm{n}$ & 1 & 4 & 5 \\
Sífilis (-), $\mathrm{n}$ & 59 & 289 & 348 \\
Total & 60 & 292 & 352 \\
\hline
\end{tabular}

$p=0,85 ; X^{2}=0,03$

\section{DISCUSSÃO}

Foram estudadas 365 pacientes gestantes em Juiz de Fora e Barbacena, em regime de pré-natal de baixo risco. Optou-se por comparar os dois serviços, considerando que poderiam ter características epidemiológicas diferentes, que podem influenciar nas frequências de doenças sexualmente transmissíveis. Ao realizar associações das variáveis em relação à origem, identificou-se que as pacientes oriundas de Juiz de Fora se declararam brancas, tinham curso superior, ganharam mais peso no pré-natal e tiveram maior número de consultas pré-natais. Quando se avaliou a escolaridade, verificou-se que as pacientes de Juiz de Fora eram aquelas que tinham maior frequência de cursos superiores. Estudo realizado em nosso meio verificou um gradiente de infecção da sífilis e sífilis congênita segundo a escolaridade materna, de forma que quanto menor a escolaridade da mulher, maior foi a ocorrência de infecção pela sífilis e de sífilis congênita, sendo encontrada um odds ratio de 16,02 (índice de confiança de 95\%: 2,76-93,01) para ensino fundamental incompleto, considerando a sífilis congênita como desfecho ${ }^{11,12}$.

A avaliação da cor foi baseada em autodeclaração e, por isso, dependeu da percepção de cada um em relação à sua cor ou raça. Apesar de alguns estudos apontarem que a sífilis está associada às mulheres pardas e pretas, neste estudo a grande maioria teve dificuldade de se declarar branca, o que poderia ser considerado um viés ${ }^{12,13}$. Não identificamos associação entre sífilis e cor da pele.

Há anos a sífilis vem crescendo no Brasil, e não existem medidas que sejam capazes de conter esse avanço. Entre os casos notificados em $2005,78 \%$ das mães realizaram pré-natal. Destas, $56 \%$ tiveram o diagnóstico de sífilis durante a gravidez e apenas $13,3 \%$ tiveram os seus parceiros tratados. Esses indicadores refletem a baixa qualidade do pré-natal no país ou até mesmo a pouca importância que os profissionais de saúde (gestores ou diretamente envolvidos no atendimento) têm dado ao diagnóstico e ao tratamento da sífilis ${ }^{14}$. Passados mais de dez anos, o Boletim Epidemiológico do Ministério da Saúde ressalta que entre os anos de 2014 e 2015, a sífilis adquirida teve um aumento de $32,7 \%$, a sífilis em gestantes $20,9 \%$ e congênita, de $19 \%$. Em outubro de 2016, o governo recomendou medidas de combate à sífilis, dentre as quais o incentivo à realização do pré-natal precoce, ainda no primeiro trimestre da gestação, a ampliação do diagnóstico por meio de teste rápido, o tratamento oportuno para a gestante e seu parceiro e o incentivo à administração de penicilina benzatina, considerada o único medicamento seguro e eficaz na prevenção da SC ${ }^{15-17}$. Nesse estudo, identificamos cinco casos de sífilis no pré-natal, que foram tratados com penicilina, e não houve nenhum caso de SC. Pode-se supor que a qualidade dos pré-natais em questão foi determinante para diagnosticar e iniciar o tratamento em tempo hábil. Nesse caso, o maior número de consultas permitiu criar uma janela de oportunidade para o tratamento.

Muitas pacientes não permitiram a realização do exame ginecológico, especialmente em Barbacena. É de extrema importância a orientação de que o exame ginecológico na gravidez não causa dano ao bebê, devendo ser realizado, além de ser uma ótima oportunidade para a coleta do exame colpocitológico. Acredita-se que as atividades de prevenção devam ser desenvolvidas, aproveitando as oportunidades do comparecimento às Unidades de Saúde. $O$ atendimento da mulher no pré-natal é um momento especial, e nele devem ser asseguradas as ações e as atividades de promoção e proteção da saúde tanto da mulher quanto de seu bebê ${ }^{18}$. Em Barbacena, houve a maior falha na realização do exame colpocitológico. Entretanto, o rastreamento em gestantes deve seguir as recomendações de periodicidade e faixa etária, como para as demais mulheres, sendo que a procura ao serviço de saúde para realização de pré-natal deve sempre ser considerada uma oportunidade para o rastreio ${ }^{18,19}$. Importante ressaltar que a sífilis é uma doença sexualmente transmissível e o exame ginecológico deve ser feito em toda gestante, até para identificar e tratar infeções genitais que possam interferir no desfecho da gravidez. Dos exames realizados, não se identificou cancro sifilítico (sífilis primária) em nenhuma paciente.

Não houve caso de infecção por HIV e hepatite $\mathrm{C}$ em ambos os serviços. A sífilis, por outro lado, foi frequente em ambos os serviços e foi associada à baixa escolaridade. Interessante avaliar que a sífilis vem aumentando nos últimos anos e, apesar de a OMS preconizar a detecção e o tratamento oportunos das gestantes e de seus parceiros sexuais portadores da sífilis, a eliminação da sífilis não aconteceu (estima-se 1 milhão de casos de sífilis por ano entre as gestantes) $)^{16,20}$. Importante ressaltar que um único caso $\mathrm{SC}$ determina falha na cadeia de 
detecção, abordagem ou tratamento de gestantes. Nas duas cidades houve casos de sífilis, sem diferença estatística. Todas as pacientes receberam tratamento com evolução satisfatória e queda do VDRL, conforme preconiza a literatura médica, e, desse modo, não houve nenhum caso de SC, o que evidencia a importância do pré-natal como rastreador de risco e capaz de mudar a história natural da doença. Entretanto, é importante ressaltar que os dois serviços estão vinculados a faculdades de medicina e, portanto, refletem uma assistência especialidade e de alto nível, o que não pode ser extrapolado para toda a rede de saúde pública. Entretanto, os dados sugerem que há necessidade de investimento em campanhas de conscientização da população, considerando que a sífilis é sexualmente transmissível e passível de prevenção. Desde março do ano passado, foi instituído no Brasil o dia de combate à sífilis, para enfatizar a importância do diagnóstico e do tratamento adequados da sífilis na gestante durante o pré-natal e da sífilis em ambos os sexos como uma doença sexualmente transmissível ${ }^{21}$. Espera-se que essas campanhas sejam capazes de reduzir os casos de sífilis no Brasil e que a sífilis congênita não seja uma realidade em nosso país em um futuro próximo.

\section{REFERÊNCIAS}

1. Zimmermmann JB, Neves HS, Souza PB, Pena DMF, Pereira MP, Nunes TR, et al. Rastreio da infecção pelo vírus da imunodeficiência humana no momento do parto. Rev Bras Ginecol Obstet. 2011;33:170-5.

2. Albuquerque GMA, Chaves EMC, Sampaio LRL. Complicações da sífilis congênita: uma revisão de literatura. Fortaleza: UFC; 2014 [citado 2017 Abr 11]. Disponível em: http:// www.repositorio.ufc.br/bitstream/ riufc/10279/1/2014_art_mcapatrocinio. $\mathrm{htm}$

3. Malvezzi CM, Sant'ana RO, Rodrigues IC, Sasaki NSGMS. Ações de controle da sífilis gestacional. São Jose do Rio Preto: UNILAGO; 2016 [citado 2017 Abr 11]. Disponível em: http://www. unilago.edu.br/revista/edicaoatual/ Sumario/2016/downloads/4.pdf

4. Domingues RMSM, Saraceni V, Hartz ZMA, Leal MC. Sífilis congênita: evento sentinela da qualidade da assistência pré-natal. Rev Saude Publica. 2013;47:37-43.

5. Domingues RMSM, Leal MC. Incidência de sífilis congênita e fatores associados à transmissão vertical da sífilis: dados do estudo Nascer no Brasil. Cad Saude Publica. 2016;32:1-12.

6. Saraceni V, Miranda AE. Relação entre a cobertura da Estratégia Saúde da Família e o diagnóstico de sífilis na gestação e sífilis congênita. Cad Saude Publica. 2012;28:490-6.

7. Federação Brasileira das Associações de Ginecologia e Obstetrícia (FEBRASGO). Ficha clínica de prénatal. São Paulo: FEBRASGO; 2008 [citado 2008 Jan 16]. Disponível em: https://pt.scribd.com/doc/316660626/ Ficha-Clinica-Pré-Natal-FEBRASGO

8. Amaral FE, Amaral PO, Andrade RVP, Resende U, Marangoni MC, Cruz R, et al. Qualidade do pré-natal: Uma comparação entre gestantes atendidas na Faculdade de Medicina de Barbacena e Universidade Federal de Juiz de Fora. Clin Biomed Res. 2016;36:124-34.

9. Federação Brasileira das Associações de Ginecologia e Obstetrícia (FEBRASGO). Assistência pré-natal. São Paulo: FEBRASGO; 2008 [citado 2017 Abr 11]. Disponível em: http:// www.itarget.com.br/newclients/ sggo.com.br/2008/extra/download/ ASSISTENCIA-PRE-NATAL

10. Brasil. Ministério da Saúde. Pré-natal e puerpério atenção qualificada e humanizada: manual técnico. Brasília: Ministério da Saúde; 2005.

11. Andrade LG, Amorim MMR, Cunha ASC, Leite SRF, Vital SA. Fatores associados à natimortalidade em uma maternidade escola em Pernambuco: estudo caso-controle. Rev Bras Ginecol Obstet. 2009;31:285-9.

12. Domingues RMSM, Leal MC. Incidência de sífilis congênita e fatores associados à transmissão vertical da sífilis: dados do estudo Nascer no Brasil. Cad Saude Publica. 2016;32:1-11.

13. Ferreira EAM. Refletindo o conceito da miscigenação no país. Guarabira: UEPB; 2012 [citado 2017 Abr 11]. Disponível em: http:// dspace.bc.uepb.edu.br:8080/xmlui/ bitstream/handle/123456789/1346/
PDF\%20-\%20Elisangela \%20 Alves\%20de\%20Morais\%20Ferreira. pdf? sequence $=1$ \&isAllowed $=y$.

14. Serviço de Vigilância Epidemiológica. Coordenação do Programa Estadual DST/Aids-SP. Coordenadoria de Controle de Doenças (CCD). Secretaria de Estado da Saúde (SES-SP). Sífilis congênita e sífilis na gestação. Rev Saude Publica. 2008;42:768-72.

15. Brasil. Governo do Brasil. Gestantes são foco de ações de combate a sífilis. Brasília; 2016 [citado 2016 Out 20]. Disponível em: http://www.brasil. gov.br/saude/2016/10/gestantes-saofoco-de-acoes-de-combate-a-sifilis

16. World Health Organization (WHO). Pregnant women must be able to access the right care at the right time, says WHO. Geneva: WHO; 2016 [citado 2017 Abr 11]. Disponível em: http://www.who.int/mediacentre/ news/releases/2016/antenatal-careguidelines/en/

17. Brasil. Ministério da Saúde. Atenção ao pré-natal de baixo risco. Brasília: Ministério da Saúde; 2012. (Cadernos de Atenção Básica; no. 32) [citado 2017 Abr 11]. Disponível em: http:// bvsms.saude.gov.br/bvs/publicacoes/ cadernos_atencao_basica_32_ prenatal.pdf

18. Instituto Nacional do Câncer (INCA). Coordenação Geral de Ações Estratégicas. Divisão de Apoio à Rede de Atenção Oncológica.Diretrizes brasileiras para o rastreamento do câncer do colo do útero. Rio de Janeiro: INCA; 2011. 
19. Yassoyama MCBM, Salomão MLM, Vicentini ME. Características das mulheres que realizam exame preventivo do colo do útero durante a gestação: bases para estratégias do Programa de Saúde da Família (PSF). Arq Ciênc Saúde. 2005;12:172-6.
20. Brasil. Secretaria de Vigilância em Saúde. Departamento de DST, Aids e Hepatites Virais. Indicadores de sífilis em gestantes. Brasília: Secretaria de Vigilância em Saúde; 2016 [citado 2016 Out 20]. Disponível em: http:// indicadoressifilis.aids.gov.br/
21. Brasil. Presidência da República. Casa Civil. Lei $n^{\circ} 13.430$, de 31 de março de 2017. Institui o dia nacional de combate à sífilis congênita. Diário Oficial da União. 2017 Abr 3 [citado 2017 Mar 31]. Disponível em: https:// www.planalto.gov.br/ccivil 03/ ato2015-2018/2017/lei//13430.htm

Recebido: Ago 21, 2017 Aceito: Mar 09, 2018 